\title{
6 Discussion
}

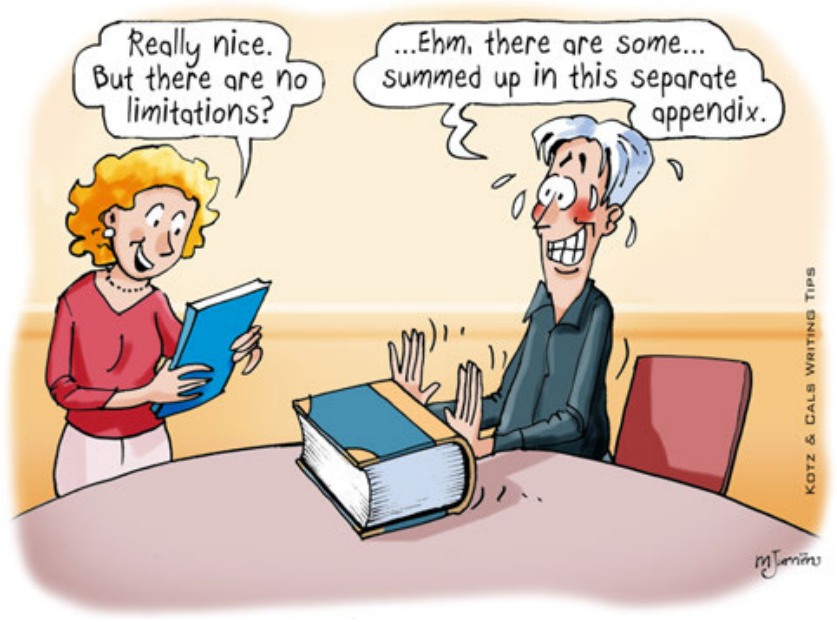

Tip 6 - Discussion: be frank in acknowledging limitations!

\section{What you should know}

The purpose of the discussion section is to give the reader a summary of the main findings and to put them into context by comparing with previous work and discussing future implications and any shortcomings of the research design. It answers the research question posed in the introduction and explains to the readers how the results support this answer.

While the structure of the introduction can be visualised as a funnel, the discussion can be visualised as an inverted funnel. Thus, the introduction and discussion together form an hourglass shape. The discussion starts with the narrowest part by answering the research question in the summary of main findings and it then gradually widens out to comparisons with other studies and the interpretation of the study findings in the wider context of the study topic. Whereas the results section merely presents data, the discussion section offers an interpretation of the data, and should never present new results. A typical discussion section consists of: main findings, comparison of findings with those reported in the literature, strengths and limitations, and implications for clinical practice and/or research. 


\section{What you should do}

Start thinking about the discussion even before collecting the first data. Many aspects and "pearls and pitfalls" of the study, as well as its relation with other studies in the field, will be discussed when developing, carrying out the research and analysing the data, and in project group meetings. Make notes and a list of keywords as a reminder of these useful discussions, while remembering your story line at all times. Do the same when presenting preliminary findings in research meetings or conferences. Questions from the audience will likely be good material for your discussion. Having such a list will greatly facilitate writing the first draft of the discussion section, and will serve as an outline for this section of the paper (see part 1: how to get started).

Start by presenting the main findings, by answering the research question in exactly the same way as you stated it in the introduction section (see part 3: introduction). If you can't present the main findings in three sentences, it may mean that you have forgotten the storyline of the paper. Do not waste words by repeating results in detail, and only use numbers or percentages if they're really necessary for your message. Don't ignore or cover up inconvenient results. Reviewers will pick them up anyway, and it weakens your paper if you try to hide them. Also, do mention unexpected findings by explicitly stating that they were unexpected and did not relate to a prior hypothesis; such honesty will strengthen your paper.

When comparing with other studies, discuss the reasons for differences and similarities with your results and do mention the limitations of those studies, but be respectful and objective. Importantly, try to stress what your data adds to the existing body of evidence.

Include a separate subsection about the strengths and weaknesses of the study. Every study has its limitations, and you should make sure to mention them. Sometimes it's possible to counterbalance a limitation with a specific strength, for instance by referring to an ancillary analysis.

End the discussion section with a conclusion presenting your findings in light of the evidence in the field and the specific strengths and limitations of your research. This is the bottom line of the paper, so try to think of it as the one-liner (citable statement) that readers (or the media) can easily understand and remember when having seen your work. Try to formulate possible implications (for clinical practice and/or research, depending on the focus of the paper). Never, ever(!), just write that further research is needed; this is practically the same as telling people not to forget to breathe. Similarly, don't try to "sell" or announce future studies, as the journal editors or its readers don't have ac- 
cess to those data at that moment. The bigger picture of your research line or $\mathrm{PhD}$ trajectory may be obvious to you, but will not interest readers of this specific paper. Embrace the uniqueness of this specific study and always remember to stick to the original storyline of the paper.

Write the discussion by imagining yourself in a dialogue with an interested reader. Depending on the scope of the journal, anticipate what kind of questions readers (and thus reviewers and editors) might have. This will help you decide what aspects deserve to get most attention, and thus the largest number of words, in your discussion. Be cautious about choosing words that are too strong. It is appropriate to use "may" or "might". "Show", "demonstrate" and "suggest" are also more appropriate than "prove", which can hardly ever be used in research.

\section{Checklist for the discussion}

- Check if the discussion has a clear inverted funnel shape with distinct sections providing:

- a summary of main findings (What did we find?);

- comparisons with other studies (What's known?, What's new? How does this fit in?);

- $\quad$ strengths and limitations (Are the findings true? Can we trust them?); and

- implications (Are the findings important? What can we do with them?).

- Answer the research question in the first paragraph and check if this is in line with the research question posed in the introduction (hourglass model)

- Check to see if the discussion section does not present new results

- Be frank about acknowledging limitations

- Ensure that the discussion offers a clear ending to the storyline of the paper

- Formulate a clear and concise conclusion as the bottom line of the paper 